Volume 136, Number 1, January 2008, Pages 193-201

S 0002-9939(07)09090-9

Article electronically published on October 11, 2007

\title{
A MÜNTZ SPACE HAVING NO COMPLEMENT IN $L_{1}$
}

\author{
IHAB AL ALAM
}

(Communicated by Nicole Tomczak-Jaegermann)

\begin{abstract}
C([0,1])$. In the present paper, we prove that there is a Müntz space not complemented in $L_{1}([0,1])$.
\end{abstract}

\section{INTRODUCTION AND NOTATION}

We denote the norm in $L_{p}([0,1])$ by $\|\cdot\|_{p}$. Suppose that $\Lambda=\left(\lambda_{i}\right)_{i=0}^{\infty}$ is a sequence of distinct positive real numbers satisfying $\sum_{i=1}^{\infty} 1 / \lambda_{i}<\infty$ and consider the closed span $[M(\Lambda)]_{L_{1}([0,1])}$ of the monomials $x^{\lambda}, \lambda \in \Lambda$. By the Müntz theorem [1, p. 172], $[M(\Lambda)]_{L_{1}[0,1]}$ is not all of $L_{1}([0,1])$, and by a later theorem of Clarkson-Erdös $[5$, p. 81] and Schwartz $[9],[M(\Lambda)]_{L_{1}([0,1])}$ consists only of "power" series $\sum_{\lambda \in \Lambda} a_{\lambda} x^{\lambda}$ convergent on $[0,1)$ if $\Lambda$ satisfies the gap condition $\inf \left\{\lambda_{i}-\lambda_{i-1}: i \in \mathbb{N}\right\}>0$.

Newman [7] gave an example of Müntz space with no complement in $C([0,1])$. In the present paper, we prove that there is a Müntz space not complemented in $L_{1}([0,1])$ (see Theorem 3.6). In Theorem 3.2, we get an $l_{1}$-Schauder decomposition of $\left[M\left(\Lambda_{\epsilon}\right)\right]_{L_{1}}$, where $\Lambda_{\epsilon}=\bigcup_{k=1}^{\infty}\left\{N_{k}, 2 N_{k}, \ldots, k N_{k}\right\}$ is not quasilacunary. But the sequence $\left\{N_{1}, N_{2}, \ldots\right\}$ of the initial terms in the blocks composing $\Lambda_{\epsilon}$ is of course lacunary, and if $\Lambda_{\epsilon}^{\prime}=\left\{N_{1}, N_{2}, \ldots\right\}$, the sequence $\left(x^{N_{k}}\right)_{k}$ is a Schauder basis of $\left[M\left(\Lambda_{\epsilon}^{\prime}\right)\right]_{L_{1}},(1+\epsilon)$-equivalent to the canonical basis of $l_{1}$, so in a sense our result contains a result due to Gurariy and Macaev [6].

To know more on the geometry of Müntz spaces, see the recent monograph of Gurariy and Lusky [5], where they prove a similar result to our Theorem 3.2 but only when $\Lambda$ is a quasilacunary sequence (cf. [5, Theorem 9.3.3]).

\section{Preliminary Results}

In this section we recall some properties of Müntz spaces that we list as propositions.

Proposition 2.1 ([1, p. 177, E.3.c]). Suppose $\Lambda=\left(\lambda_{i}\right)_{i=0}^{\infty}$ is a sequence of positive real numbers satisfying the gap condition

$$
\inf \left\{\lambda_{i}-\lambda_{i-1}: i \in \mathbb{N}\right\}>0 \quad \text { and } \quad \sum_{i=1}^{\infty} \frac{1}{\lambda_{i}}<\infty .
$$

Received by the editors October 10, 2006.

2000 Mathematics Subject Classification. Primary 41A10, 41A17; Secondary 46B20, 46E15.

Key words and phrases. Müntz spaces, complementation, Schauder basis. 
Then, for every $\epsilon>0$, there is a constant $c(\epsilon, \Lambda)$ depending only on $\epsilon$ and $\left(\lambda_{i}\right)_{i=0}^{\infty}$ such that

$$
\left|a_{i}\right|(1-\epsilon)^{\lambda_{i}} \leq c(\epsilon, \Lambda)\|p\|_{2}
$$

for every $p \in \operatorname{span}\left\{x^{\lambda_{0}}, x^{\lambda_{1}}, \ldots\right\}$ of the form $p(x)=\sum_{i=0}^{n} a_{i} x^{\lambda_{i}}$.

Proposition 2.2 ([1, p. 185, E.8.a]). Assume that $0 \leq \lambda_{0}<\lambda_{1}<\ldots$ with $\sum_{i=1}^{\infty} 1 / \lambda_{i}<\infty$. Then, for every $\epsilon \in(0,1)$, there is a constant $\gamma(\epsilon, \Lambda)$ depending only on $\epsilon$ and $\left(\lambda_{i}\right)_{i=0}^{\infty}$ such that

$$
\|p\|_{[0,1-\epsilon]} \leq \gamma(\epsilon, \Lambda) \int_{1-\epsilon}^{1}|p(x)| d x
$$

for every $p \in \operatorname{span}\left\{x^{\lambda_{0}}, x^{\lambda_{1}}, \ldots\right\}$.

Proposition 2.3 (Newman's inequality in $L_{p}$, [1, p. 279]). Let $p \in[1, \infty)$. If $\Lambda=$ $\left(\lambda_{i}\right)_{i=0}^{\infty}$ is a sequence of distinct positive real numbers greater than $-1 / p$, then

$$
\left\|x P^{\prime}\right\|_{p} \leq\left(\frac{1}{p}+12 \sum_{j=0}^{n}\left(\lambda_{j}+\frac{1}{p}\right)\right)\|P\|_{p}
$$

for every $P \in M_{n}(\Lambda)=\operatorname{span}\left\{x^{\lambda_{0}}, x^{\lambda_{1}}, \ldots, x^{\lambda_{n}}\right\}$.

In Müntz spaces, we obtain better estimates.

Remark 2.4. Let $\Lambda=\left(\lambda_{i}\right)_{i=0}^{\infty}$ be a sequence of distinct positive integers satisfying $\sum_{i=1}^{\infty} 1 / \lambda_{i}<\infty$. Then by Proposition 2.2 there exists a constant $C(\Lambda)>0$ (depending only on $\Lambda$ ) with

$$
\left\|p^{\prime}\right\|_{1} \leq C(\Lambda)\left(\sum_{j=m}^{n} \lambda_{j}\right)\|p\|_{1}
$$

whenever $p(x)=\sum_{j=m}^{n} \alpha_{j} x^{\lambda_{j}}$ (for any $m$ and $n$ ).

The next corollary is a direct consequence of the two previous propositions.

Corollary 2.5. Let $\Lambda=\left(\lambda_{i}\right)_{i=0}^{\infty}$ be a sequence of distinct positive integers satisfying $\sum_{\text {that }}^{\infty} 1 / \lambda_{i}<\infty$. Then there is a constant $K(\Lambda)>0$ (depending only on $\Lambda$ ) such

$$
\|p\|_{\infty} \leq K(\Lambda)\left(1+\sum_{j=m}^{n} \lambda_{j}\right)\|p\|_{1}
$$

whenever $p(x)=\sum_{j=m}^{n} \alpha_{j} x^{\lambda_{j}}$ (for any $m$ and $\left.n\right)$.

Proof. Writing $p(x)=p(0)+\int_{0}^{x} p^{\prime}(t) d t$, we have $|p(x)| \leq|p(0)|+\int_{0}^{1}\left|p^{\prime}(t)\right| d t$. By Proposition 2.2 there is $\gamma(\Lambda)$ such that $|p(0)| \leq \gamma(\Lambda) \int_{0}^{1}|p(t)| d t$. Newman's 
inequality applied to $p$ yields $\left\|p^{\prime}\right\|_{1} \leq C(\Lambda)\left(\sum_{j=m}^{n} \lambda_{j}\right)\|p\|_{1}$. Then

$$
\begin{aligned}
\|p\|_{\infty} & \leq \gamma(\Lambda)\|p\|_{1}+C(\Lambda)\left(\sum_{j=m}^{n} \lambda_{j}\right)\|p\|_{1} \\
& \leq \max (\gamma(\Lambda), C(\Lambda))\left(1+\sum_{j=m}^{n} \lambda_{j}\right)\|p\|_{1} .
\end{aligned}
$$

We take $K(\Lambda)=\max (\gamma(\Lambda), C(\Lambda))$.

\section{MAin RESUltS}

The next lemma is the key point of our construction.

Lemma 3.1. For every $\epsilon>0$ and every $m \in \mathbb{N}$, there is a positive integer $N=$ $N(\epsilon, m)$ depending only on $\epsilon$ and $m$ (but not on $p$ or $q$ ) such that

$$
\|p(x)\|_{1}+\left\|q\left(x^{N}\right)\right\|_{1} \leq(1+\epsilon)\left\|p(x)+q\left(x^{N}\right)\right\|_{1}
$$

for every $p, q \in \operatorname{span}\left\{1, x, x^{2}, \ldots, x^{m}\right\}$ with $q(0)=0$.

Proof. Let $\left(N_{k}\right)_{k=1}^{\infty}$ be an increasing sequence of positive integers satisfying $N_{1}>m$ and $N_{k+1}>k N_{k}$. Put $\Lambda_{m}=\{1,2, \ldots, m\} \cup \bigcup_{k=1}^{\infty}\left\{N_{k}, 2 N_{k}, \ldots, k N_{k}\right\}$.

Let $p, q \in \operatorname{span}\left\{1, x, x^{2}, \ldots, x^{m}\right\}$ with $q(x)=\sum_{j=1}^{m} q_{j} x^{j}$. Then for any $\alpha \in(0,1)$ and any $N \in\left\{N_{m}, N_{m+1}, \ldots\right\}$, we have

$$
\begin{aligned}
\int_{0}^{1-\alpha}\left|q\left(x^{N}\right)\right| d x & \leq \sum_{j=1}^{m}\left|q_{j}\right| \int_{0}^{1-\alpha} x^{j N} d x \\
& \leq \frac{m}{N} \sup _{1 \leq j \leq m}\left|q_{j}\right|(1-\alpha)^{j N} \\
& \leq c(\alpha, m) \frac{m}{N}\left\|p(x)+q\left(x^{N}\right)\right\|_{2} .
\end{aligned}
$$

The last inequality follows from the fact that $q_{j}$ is the coefficient of $x^{j N}$ in $p(x)+$ $q\left(x^{N}\right) \in M\left(\Lambda_{m}\right)$ and from Proposition 2.1. Therefore

$$
\int_{0}^{1-\alpha}\left|q\left(x^{N}\right)\right| d x \leq c(\alpha, m) \frac{m}{N}\left\|p(x)+q\left(x^{N}\right)\right\|_{\infty}^{\frac{1}{2}}\left\|p(x)+q\left(x^{N}\right)\right\|_{1}^{\frac{1}{2}} .
$$

On the other hand, writing $Q(x)=p(x)+q\left(x^{N}\right), Q(x) \in M\left(\Lambda_{m}\right)$. Then we have by Corollary 2.5

$$
\|Q\|_{\infty} \leq K\left(\Lambda_{m}\right)\left(1+\sum_{j=1}^{m} j+N \sum_{j=1}^{m} j\right)\|Q\|_{1} \leq K\left(\Lambda_{m}\right) m^{2} N\|Q\|_{1}
$$

and hence, if we put $\delta(\alpha, m)=m^{2} c(\alpha, m) \sqrt{K\left(\Lambda_{m}\right)}$, we have

$$
\int_{0}^{1-\alpha}\left|q\left(x^{N}\right)\right| d x \leq \frac{\delta(\alpha, m)}{\sqrt{N}}\left\|p(x)+q\left(x^{N}\right)\right\|_{1}
$$


In the same way, by Corollary 2.5 we have

$$
\|p\|_{\infty} \leq K\left(\Lambda_{m}\right)\left(1+\sum_{j=0}^{m} j\right)\|p\|_{1} \leq m^{2} K\left(\Lambda_{m}\right)\|p\|_{1},
$$

and so we have

$$
\int_{1-\alpha}^{1}|p(x)| d x \leq \alpha\|p\|_{\infty} \leq \alpha m^{2} K\left(\Lambda_{m}\right)\|p\|_{1} .
$$

Now we return to the proof of our inequality. We can write

$$
\begin{gathered}
\int_{0}^{1}\left|p(x)+q\left(x^{N}\right)\right| d x \\
\geq\left(\int_{0}^{1-\alpha}|p(x)| d x-\int_{0}^{1-\alpha}\left|q\left(x^{N}\right)\right| d x\right)+\left(\int_{1-\alpha}^{1}\left|q\left(x^{N}\right)\right| d x-\int_{1-\alpha}^{1}|p(x)| d x\right) \\
\geq \int_{0}^{1}|p(x)| d x+\int_{0}^{1}\left|q\left(x^{N}\right)\right| d x-2 \int_{1-\alpha}^{1}|p(x)| d x-2 \int_{0}^{1-\alpha}\left|q\left(x^{N}\right)\right| d x .
\end{gathered}
$$

$\mathrm{U} \operatorname{sing}(*)$ and $(* *)$, we obtain that

$$
\begin{gathered}
\int_{0}^{1}\left|p(x)+q\left(x^{N}\right)\right| d x \\
\geq\left(1-2 \alpha m^{2} K\left(\Lambda_{m}\right)\right)\left(\|p\|_{1}+\left\|q\left(x^{N}\right)\right\|_{1}\right)-2 \frac{\delta(\alpha, m)}{\sqrt{N}}\left\|p(x)+q\left(x^{N}\right)\right\|_{1},
\end{gathered}
$$

and hence

$$
\|p(x)\|_{1}+\left\|q\left(x^{N}\right)\right\|_{1} \leq\left(\frac{1+2 \frac{\delta(\alpha, m)}{\sqrt{N}}}{1-2 \alpha m^{2} K\left(\Lambda_{m}\right)}\right)\left\|p(x)+q\left(x^{N}\right)\right\|_{1} .
$$

Suppose $\alpha$ is such that $2 \alpha m^{2} K\left(\Lambda_{m}\right) \leq \frac{\epsilon}{2+\epsilon}$ and $N \in\left\{N_{m}, N_{m+1}, \ldots\right\}$ such that $2 \frac{\delta(\alpha, m)}{\sqrt{N}} \leq \frac{\epsilon}{2+\epsilon}$. We have $\|p(x)\|_{1}+\left\|q\left(x^{N}\right)\right\|_{1} \leq(1+\epsilon)\left\|p(x)+q\left(x^{N}\right)\right\|_{1}$.

In the following theorem, we get an $l_{1}$-Schauder decomposition of $\left[M\left(\Lambda_{\epsilon}\right)\right]_{L_{1}}$, where $\Lambda_{\epsilon}=\bigcup_{k=1}^{\infty}\left\{N_{k}, 2 N_{k}, \ldots, k N_{k}\right\}$ is not quasilacunary $\left(\frac{k N_{k}}{(k-1) N_{k}} \rightarrow 1\right)$. But, for every set with shape $\Lambda_{\epsilon}^{\prime}=\bigcup_{k=1}^{\infty} A_{k}$, where $A_{k} \subset\left\{N_{k}, 2 N_{k}, \ldots, k N_{k}\right\}$ with $\max _{k} \operatorname{card} A_{k}<\infty, \Lambda_{\epsilon}^{\prime}$ is quasilacunary and $\left[M\left(\Lambda_{\epsilon}^{\prime}\right)\right]_{L_{1}}$ is isomorphic to $l_{1}$. Obviously, the sequence $\left\{N_{1}, N_{2}, \ldots\right\}$ of the initial terms in the blocks composing $\Lambda_{\epsilon}$ is lacunary $\left(N_{k+1}>k N_{k}\right)$. Writing $\Lambda_{\epsilon}^{\prime \prime}=\left\{N_{1}, N_{2}, \ldots\right\}$, the sequence $\left(x^{N_{k}}\right)_{k}$ is a Schauder basis of $\left[M\left(\Lambda_{\epsilon}^{\prime \prime}\right)\right]_{L_{1}},(1+\epsilon)$-equivalent to the canonical basis of $l_{1}$, so in a sense our result contains the theorem of Gurariy and Lusky [5, Theorem 9.3.3].

Theorem 3.2. For every $\epsilon>0$, there exists a sequence of increasing positive integers, $\left(N_{k}\right)_{k=1}^{\infty}$ with $N_{k+1}>k N_{k}$, depending only on $\epsilon$ such that if $\Lambda_{\epsilon}=\bigcup_{k=1}^{\infty}\left\{N_{k}, 2 N_{k}, \ldots, k N_{k}\right\}$, we have $\left[M\left(\Lambda_{\epsilon}\right)\right]_{L_{1}} \equiv\left(\sum_{k=1}^{\infty} F_{k}\right)_{\left(l_{1}\right)}$ where $F_{k}=\operatorname{sp}\left\{x^{N_{k}}, x^{2 N_{k}}, \ldots, x^{k N_{k}}\right\}$. More precisely, we have $\sum_{k=1}^{\infty}\left\|p_{k}\left(x^{N_{k}}\right)\right\|_{1} \leq$ $(1+\epsilon)\|f\|_{1}$ whenever $f \in\left[M\left(\Lambda_{\epsilon}\right)\right]_{L_{1}}$ with an Erdös decomposition:

$$
f(x)=\sum_{k=1}^{\infty} p_{k}\left(x^{N_{k}}\right), \quad x \in[0,1), \quad p_{k} \in \operatorname{span}\left\{1, x, x^{2}, \ldots, x^{k}\right\} .
$$


Proof. Let $\epsilon_{k}>0, k=1,2, \ldots$, be such that $\prod_{k=1}^{\infty}\left(1+\epsilon_{k}\right)=(1+\epsilon)$. Now we fix $N_{1} \geq 0$ and define $N_{k+1}=N\left(\epsilon_{k+1}, k N_{k}\right)$ as in the preceding lemma.

We shall use induction on $n$ to prove

$$
\sum_{k=1}^{n}\left\|p_{k}\left(x^{N_{k}}\right)\right\|_{1} \leq \prod_{k=1}^{n}\left(1+\epsilon_{k}\right)\left\|\sum_{k=1}^{n} p_{k}\left(x^{N_{k}}\right)\right\|_{1}
$$

for every $n$ and every $p_{k} \in \operatorname{span}\left\{1, x, x^{2}, \ldots, x^{k}\right\}$. The result is trivial for $n=1$. Now we may assume that it holds for $n-1$, that is to say

$$
\sum_{k=1}^{n-1}\left\|p_{k}\left(x^{N_{k}}\right)\right\|_{1} \leq \prod_{k=1}^{n-1}\left(1+\epsilon_{k}\right)\left\|\sum_{k=1}^{n-1} p_{k}\left(x^{N_{k}}\right)\right\|_{1} .
$$

We now apply Lemma 3.1 with

$$
p(x)=\sum_{k=1}^{n-1} p_{k}\left(x^{N_{k}}\right), \quad q(x)=p_{n}(x) \quad \text { and } \quad m=(n-1) N_{n-1} .
$$

The degrees of $p$ and $q$ are clearly less than $m$, so the hypotheses of Lemma 3.1 are satisfied. Then

$$
\begin{aligned}
\sum_{k=1}^{n}\left\|p_{k}\left(x^{N_{k}}\right)\right\|_{1} & \leq \prod_{k=1}^{n-1}\left(1+\epsilon_{k}\right)\left\|\sum_{k=1}^{n-1} p_{k}\left(x^{N_{k}}\right)\right\|_{1}+\left\|p_{n}\left(x^{N_{n}}\right)\right\|_{1} \\
& \leq \prod_{k=1}^{n}\left(1+\epsilon_{k}\right)\left\|\sum_{k=1}^{n} p_{k}\left(x^{N_{k}}\right)\right\|_{1}
\end{aligned}
$$

and the induction is complete. Now, let $f(x)=\sum_{k=1}^{\infty} p_{k}\left(x^{N_{k}}\right) \in\left[M\left(\Lambda_{\epsilon}\right)\right]_{L_{1}}$, where $p_{k} \in \operatorname{span}\left\{1, x, x^{2}, \ldots, x^{k}\right\}$. Fix $\rho \in(0,1)$, and let $f_{\rho}(x)=f(\rho x) \in\left[M\left(\Lambda_{\epsilon}\right)\right]_{L_{1}}$. By (1), we have for every $n \geq 1$

$$
\sum_{k=1}^{n}\left\|p_{k}\left((\rho x)^{N_{k}}\right)\right\|_{1} \leq(1+\epsilon)\left\|\sum_{k=1}^{n} p_{k}\left((\rho x)^{N_{k}}\right)\right\|_{1} .
$$

We may now let $n \rightarrow \infty$ to get $\sum_{k=1}^{\infty}\left\|p_{k}\left((\rho x)^{N_{k}}\right)\right\|_{1} \leq(1+\epsilon)\left\|f_{\rho}\right\|_{1}$. Then letting $\rho \rightarrow 1$, gives $\sum_{k=1}^{\infty}\left\|p_{k}\left(x^{N_{k}}\right)\right\|_{1} \leq(1+\epsilon)\|f\|_{1}$. This ends the proof of Theorem 3.2 .

Remark 3.3. For $\epsilon>0$, let $\Lambda_{\epsilon}=\left(N_{k}\right)_{k}$ given by the preceding theorem. We have that $\left[M\left(\Lambda_{\epsilon}\right)\right]_{L_{1}}$ is $(1+\epsilon)$-isomorphic to $l_{1}$ so by choosing $\epsilon$ such that $(1+\epsilon) \leq \sqrt{2}$, we have, by $\left[2\right.$, Theorem A], that $\left[M\left(\Lambda_{\epsilon}\right)\right]_{L_{1}}$ is complemented in $L_{1}([0,1])$.

We will need the following:

Theorem 3.4 ([3, Theorem 1.1]). There is a $c>0$ such that any projection from $L_{1}([-1,1])$ onto the span of $1, x, x^{2}, \ldots, x^{n}$ has norm $>c \log n$.

Note that this theorem remains true if one replaces $L_{1}([-1,1])$ by $L_{1}([0,1])$, because the isometry $\theta: L_{1}([-1,1]) \mapsto L_{1}([0,1])$ taking $f(x)$ into $2 f(2 x-1)$ transforms sp $\left\{1, x, x^{2}, \ldots, x^{n}\right\}$ onto itself.

Lemma 3.5. There is a $c>0$ such that for any $N \geq 1$ and any projection $P$ from $L_{1}([0,1])$ onto $\operatorname{sp}\left\{x^{N-1}, x^{2 N-1}, \ldots, x^{k N-1}\right\}$, we have $\|P\|>c \log k$. 
Proof. Let $P$ be such a projection and let $T_{N}$ denote the map

$$
\begin{aligned}
T_{N}: L_{1}([0,1]) & \mapsto L_{1}([0,1]) \\
f(x) & \mapsto N x^{N-1} f\left(x^{N}\right) .
\end{aligned}
$$

Here $T_{N}$ is a surjective isometry with $T_{N}^{-1}(f)(x)=\frac{1}{N} x^{\frac{1}{N}-1} f\left(x^{\frac{1}{N}}\right)$ for $x \in(0,1]$, and $T_{N}\left(\operatorname{sp}\left\{1, x, \ldots, x^{k-1}\right\}\right)=s p\left\{x^{N-1}, x^{2 N-1}, \ldots, x^{k N-1}\right\}$. Then $T_{N}^{-1} P T_{N}$ is a projection from $L_{1}([0,1])$ onto the span of $\left\{1, x, x^{2}, \ldots, x^{k-1}\right\}$. Theorem 3.4 implies that $\left\|T_{N}^{-1} P T_{N}\right\| \geq c \log (k-1)$. Finally, since $T_{N}$ is an isometry, we see that $\|P\|=\left\|T_{N}^{-1} P T_{N}\right\|$ and the lemma is established.

The following theorem is the main result of the paper.

Theorem 3.6. There is an infinite dimensional Müntz space that is isomorphic to $\left(\sum_{k=0}^{\infty} R_{k}\right)_{\left(l_{1}\right)}$ and not complemented in $L_{1}[0,1]$.

Proof. Let $\Lambda_{\epsilon}=\bigcup_{k=1}^{\infty}\left\{N_{k}, 2 N_{k}, \ldots, k N_{k}\right\}$ be given by Theorem 3.2 and $\Lambda^{\prime}=$ $\bigcup_{k=1}^{\infty}\left\{N_{k}-1,2 N_{k}-1, \ldots, k N_{k}-1\right\}$. By Proposition 2.2 the map

$$
\begin{aligned}
T:\left[M\left(\Lambda^{\prime}\right)\right]_{L_{1}} & \mapsto\left[M\left(\Lambda_{\epsilon}\right)\right]_{L_{1}} \\
f(x) & \mapsto x f(x)
\end{aligned}
$$

is a surjective isomorphism. So by Theorem 3.2 and the proof of Lemma 3.5 we have that $\left[M\left(\Lambda^{\prime}\right)\right]_{L_{1}}$ is isomorphic to $\left(\sum_{k=0}^{\infty} R_{k}\right)_{\left(l_{1}\right)}\left(R_{k}=\operatorname{span}\left\{1, x, x^{2}, \ldots, x^{k}\right\}\right)$, and then, if $\Pi_{k}$ is the map defined by $\Pi_{k}(f)=x^{-1} p_{k}\left(x^{N_{k}}\right)$, where $f(x)=$ $\sum_{j=1}^{\infty} x^{-1} p_{j}\left(x^{N_{j}}\right) \in\left[M\left(\Lambda^{\prime}\right)\right]_{L_{1}}$ (its decomposition of Erdös), we have $\left\|\Pi_{k}\right\| \leq c$ independently of $k$. The map $\Pi_{k}$, however, is a projection from $\left[M\left(\Lambda^{\prime}\right)\right]_{L_{1}}$ onto $F_{k}=\operatorname{sp}\left\{x^{N_{k}-1}, x^{2 N_{k}-1}, \ldots, x^{k N_{k}-1}\right\}$. Thus if we had a bounded projection $P$ from $L_{1}([0,1])$ onto $\left[M\left(\Lambda^{\prime}\right)\right]_{L_{1}[0,1]}$, then $\Pi_{k} P$ would be a projection from $L_{1}[0,1]$ onto sp $\left\{x^{N_{k}-1}, x^{2 N_{k}-1}, \ldots, x^{k N_{k}-1}\right\}$. The bound $\left\|\Pi_{k} P\right\| \leq c\|P\|$ would therefore give a contradiction to Lemma 3.5 !

3.1. Sequences equivalent to canonical $l_{1}$-bases. Here we give another application of Lemma 3.5. We construct a Müntz space that has a basis equivalent to the natural basis in $l_{1}$. First we need the following important technical lemma.

Lemma 3.7. Let $k$ be a positive integer, and let $X_{k}=s p\left\{x^{k-1}, x^{k}\right\} \subset L_{1}([0,1])$. Then the constant of the basis $\left(x^{k-1}, x^{k}-x^{k-1}\right)$ is bounded independently of $k$, and so $\left(x^{k-1}, x^{k}-x^{k-1}\right)_{k}$ is a basis of the space $\left(\sum_{k=1}^{\infty} s p\left\{x^{k-1}, x^{k}\right\}\right)_{\left(l_{1}\right)}$ equivalent to the natural basis in $l_{1}$.

Proof. It is sufficient to prove that for some $C>0$, independent of $k$, and for an arbitrary $a, b \in \mathbb{C}$, we have the following inequality:

$$
\left\|a x^{k-1}\right\|_{1} \leq C\left\|a x^{k-1}+b\left(x^{k}-x^{k-1}\right)\right\|_{1} .
$$

Without loss of generality we may assume that $a=1$ and $b>0$. Let $f(x)=$ $x^{k-1}+b\left(x^{k}-x^{k-1}\right)=(1-b) x^{k-1}+b x^{k}$. To estimate $\|f\|_{1}$, we consider two cases:

(1) $b \in] 0,1]$, which implies that $f(x)>0$, if $x \in[0,1]$. Then

$$
\|f\|_{1}=\int_{0}^{1} f(x) d x=\frac{1-b}{k}+\frac{b}{k+1} \geq \frac{1}{k}-\frac{1}{k^{2}} \geq \frac{1}{2}\left\|x^{k-1}\right\|_{1} .
$$


(2) $b>1, f(x)=0$ implies $\left.x=\frac{b-1}{b} \in\right] 0,1[$. Then

$$
\begin{aligned}
\|f\|_{1} & =-\int_{0}^{\frac{b-1}{b}} f(x) d x+\int_{\frac{b-1}{b}}^{1} f(x) d x \\
& =\frac{1}{k(k+1)}\left(2 b\left(1-\frac{1}{b}\right)^{k+1}-b+k+1\right) .
\end{aligned}
$$

It is sufficient to prove that there is a constant $C \in] 0,1[$ such that

$(* * *)$

$$
2 b\left(1-\frac{1}{b}\right)^{k+1}-b+k \geq C k
$$

(the constant $\mathrm{C}$ will be chosen below).

Let $X=1-\frac{1}{b} \in[0,1]$. Then $(* * *)$ is equivalent to

$$
\begin{gathered}
p(X)=2 X^{k+1}+(C-1) k X+(1-C) k-1 \geq 0 \quad \text { if } X \in[0,1] \\
\left.p^{\prime}(X)=2(k+1) X^{k}+(C-1) k=0 \text { implies } X=\left(\frac{1-C}{2} \frac{k}{k+1}\right)^{\frac{1}{k}} \in\right] 0,1[.
\end{gathered}
$$

Notice that $p(0)=(1-C) k>0$ and $p(1)=1>0$. Therefore it is sufficient to prove that

$$
\begin{gathered}
p\left(\left(\frac{1-C}{2} \frac{k}{k+1}\right)^{\frac{1}{k}}\right)>0 \\
p\left(\left(\frac{1-C}{2} \frac{k}{k+1}\right)^{\frac{1}{k}}\right)=(1-C)\left[\left(\frac{1-C}{2}\right)^{\frac{1}{k}}\left(\frac{k}{k+1}\right)^{\frac{k+1}{k}}-k\left(\frac{1-C}{2}\right)^{\frac{1}{k}}\left(\frac{k}{k+1}\right)^{\frac{1}{k}}+k\right]-1 .
\end{gathered}
$$

Writing $\left(\frac{1-C}{2}\right)^{\frac{1}{k}}=1+\frac{1}{k} \ln \frac{1-C}{2}+o\left(\frac{1}{k}\right)$, we have for large enough $k$,

$$
p\left(\left(\frac{1-C}{2} \frac{k}{k+1}\right)^{\frac{1}{k}}\right) \approx_{k}(1-C)\left[1+\ln \frac{2}{1-C}\right]-1 .
$$

Now choose $C \in] 0,1\left[\operatorname{such}\right.$ that $(1-C)\left[1+\ln \frac{2}{1-C}\right]>1$, for example $C=\frac{1}{2}$ and the lemma is established.

Gurariy and Macaev [6] proved that $\left(x^{\lambda}\right)_{\lambda \in \Lambda}$ is a basis of $[M(\Lambda)]_{L_{1}}$, equivalent to the natural basis in $l_{1}$ if and only if $\Lambda$ is lacunary. In the next proposition we give a basis equivalent to the natural basis in $l_{1}$ for some Müntz spaces $[M(\Lambda)]_{L_{1}}$, where $\Lambda$ is not lacunary.

Proposition 3.8. Let $\left(N_{k}\right)_{k=1}^{\infty}$ be defined by $N_{k+1}=N\left(\epsilon_{k+1}, k N_{k}\right.$ ) (as in the proof of Theorem 3.2), where $\prod_{k=1}^{\infty}\left(1+\epsilon_{k}\right)=(1+\epsilon)$ and $\Lambda=N_{1} \cup \bigcup_{k=2}^{\infty}\left\{(k-1) N_{k}, k N_{k}\right\}$. Then $[M(\Lambda)]_{L_{1}}$ is isomorphic to $l_{1}$ and the sequence

$$
x^{N_{1}},\left(x^{(k-1) N_{k}}, x^{k N_{k}}-x^{(k-1) N_{k}}\right)_{k=2}^{\infty}
$$

is a basis of $[M(\Lambda)]_{L_{1}}$, equivalent to the natural basis in $l_{1}$.

Proof. Let $T_{N_{k}}$ denote the map

$$
\begin{aligned}
T_{N_{k}}: L_{1}([0,1]) & \mapsto L_{1}([0,1]) \\
f(x) & \mapsto N_{k} x^{N_{k}-1} f\left(x^{N_{k}}\right) .
\end{aligned}
$$

$T_{N_{k}}$ is a surjective isometry with

$$
T_{N_{k}}\left(\operatorname{span}\left\{x^{k-1}, x^{k}\right\}\right)=\operatorname{span}\left\{x^{k N_{k}-1}, x^{(k+1) N_{k}-1}\right\} .
$$


Let

$$
X=\left(\operatorname{span}\{1\}+\sum_{k=1}^{\infty} \operatorname{span}\left\{x^{k-1}, x^{k}\right\}\right)_{\left(l_{1}\right)}
$$

and

$$
Y=\overline{\operatorname{span}}^{\|\cdot\|_{1}}\left\{x^{N_{1}-1}, x^{(k-1) N_{k}-1}, x^{k N_{k}-1} ; k \geq 2\right\} .
$$

Define $T$ by

$$
\begin{aligned}
& T: X \mapsto Y \\
&\left(p_{k}\right)_{k=0}^{\infty} \mapsto \sum_{k=1}^{\infty} T_{N_{k}}\left(p_{k-1}\right) .
\end{aligned}
$$

By Theorem 3.2, $T$ is an isomorphism, and by Proposition 2.2, $x T$ is an isomorphism from $\left(s p\{1\}+\sum_{k=1}^{\infty} s p\left\{x^{k-1}, x^{k}\right\}\right)_{\left(l_{1}\right)}$ onto $[M(\Lambda)]_{L_{1}}$ and hence by Lemma 3.7 , the sequence $x^{N_{1}},\left(x^{(k-1) N_{k}}, x^{k N_{k}}-x^{(k-1) N_{k}}\right)_{k=2}^{\infty}$ is a basis of $[M(\Lambda)]_{L_{1}}$ equivalent to the natural basis in $l_{1}$.

\section{Complemented Müntz spaces isomorphic to $l_{1}$}

A family $F=\left\{f_{j}: j \in \mathbb{N}\right\}$ of $L_{1}([0,1])$ functions is called relatively disjoint if $\sup _{j \in \mathbb{N}}\left\|f_{j}\right\|_{1}<\infty$ and there exist constants $0<\epsilon<\delta$ and disjoint measurable sets $E_{j} \subset[0,1]$ such that

$$
\int_{E_{j}}\left|f_{j}(x)\right| d x>\delta \quad \text { and } \quad \sum_{i=1, i \neq j}^{\infty} \int_{E_{i}}\left|f_{j}(x)\right| d x<\epsilon, \quad j \in \mathbb{N} .
$$

Lemma 4.1 ([8, Prop.3.1]). A relatively disjoint family $F$ of $L_{1}([0,1])$ functions is equivalent to the natural basis in $l_{1}$, and $\overline{\operatorname{span}}(F)$ is complemented in $L_{1}([0,1])$.

The following result was already proved when $\lambda_{j}=m^{j}, m \geq 8$ (see [4, Lemma 11]). We actually have a slightly better result.

Theorem 4.2. Suppose $\Lambda=\left(\lambda_{j}\right)_{j=0}^{\infty}$ is a sequence of positive real numbers satisfying $\lambda_{j+1} / \lambda_{j} \geq r \geq 4.5$. Then there is $j_{0}>0$ such that $\left\{\left(\lambda_{j}+1\right) x^{\lambda_{j}}: j>j_{0}\right\}$ is a relatively disjoint family of $L_{1}([0,1])$ and hence $[M(\Lambda)]_{L_{1}}$ is complemented in $L_{1}([0,1])$.

Proof. Let $E_{j}=\left(e^{-\frac{2}{\lambda_{j}}}, e^{-\frac{2}{r \lambda_{j}}}\right)$. Then

$$
\int_{E_{j}}\left(\lambda_{j}+1\right) x^{\lambda_{j}} d x=e^{-\frac{2\left(\lambda_{j}+1\right)}{r \lambda_{j}}}-e^{-\frac{2\left(\lambda_{j}+1\right)}{\lambda_{j}}} \geq e^{\frac{2\left(\lambda_{j}+1\right)}{r \lambda_{j}}}-e^{-2} \underset{j \rightarrow+\infty}{\longrightarrow} e^{-\frac{2}{r}}-e^{-2} .
$$

Moreover $\left.e^{-\frac{2}{r}}-e^{-2} \in\right] \frac{1}{2}, 1[$; therefore there exist $\delta \in] \frac{1}{2}, 1\left[\right.$ and $j_{0}>0$ such that for any $j>j_{0}$, we have

$$
\int_{E_{j}}\left(\lambda_{j}+1\right) x^{\lambda_{j}} d x>\delta \quad \text { and } \quad \sum_{i=1, i \neq j}^{\infty} \int_{E_{i}}\left(\lambda_{j}+1\right) x^{\lambda_{j}} d x<1-\delta .
$$

Choose $\epsilon=1-\delta$ so $\left\{\left(\lambda_{j}+1\right) x^{\lambda_{j}}: j>j_{0}\right\}$ is $(\delta, \epsilon)$-relatively disjoint and then using Lemma 4.1, $[M(\Lambda)]_{L_{1}}$ is complemented in $L_{1}([0,1])$. 


\section{ACKNowledgments}

The author expresses his sincere thanks to his supervisor, Professor Pascal Lefevre, who gave him helpful advice and introduced him to the geometry of Müntz spaces, which motivated the present work.

\section{REFERENCES}

1. P. Borwein and T. Erdélyi, Polynomials and polynomial inequalities, Springer, Berlin, Heidelberg, New York, 1995. MR1367960 (97e:41001)

2. L. E. Dor, On projection in $L_{1}$, Ann. of Math. (2) 102 (1975), no. 3, 463-474. MR0420244 (54:8258)

3. E. Görlich and A.P. Rohs, Asymptotic bounds for projection operators on lebesgue spaces, Integral Transforms Spec. Funct. 3 (1995), no. 2, 85-98. MR1410597 (97h:41011)

4. E. Görlich and A.P. Rohs, Bounds for relative projection constants in $L^{1}(-1,1)$, Constr. Approx. 14 (1998), no. 4, 589-597. MR1646559 (99h:41009)

5. V. I. Gurariy and W. Lusky, Geometry of Müntz spaces and related questions, Lecture Notes in Mathematics, Springer, Berlin, Heidelberg, New York, 2005. MR2190706

6. V. I. Gurariy and V. Macaev, Lacunary power sequences in $C$ and $L_{p}$, Izvestiya Acad. Nauk SSSR, 30 (1966), 3-14. MR0190703 (32:8115)

7. D. J. Newman, A Müntz space having no complement, J. Approx. Theory 40 (1984), 351-354. MR740647 (85g:41015)

8. H.P. Rosenthal, On relatively disjoint families of measures, with some applications to Banach space theory, Studia Math. 37 (1973), 13-36. MR0270122 (42:5015)

9. L. Schwartz, Étude des sommes d'exponentielles réelles, Act. Sci. Ind., no. 959, Hermann et Cie, Paris, 1943, 89 pp. MR0014502 (7:294c)

Université des Sciences et Technologies de Lille, Laboratoire Paul Painlevé U.M.R. CNRS 8524, U.F.R. De Mathématiques, 59655 Villeneuve D'Ascq Cedex, France

E-mail address: Ihab.Alalam@math.univ-lille1.fr 